\title{
Diagnostic Role of Ultrasonography in Obstetrics and Gynaecology
}

\author{
Ziban Nahar ${ }^{1}$, A H M Tohurul Islam ${ }^{2}$, N Atia Lovely ${ }^{3}$, M Hafizur Rahman ${ }^{4}$
}

\begin{abstract}
Ultrasonography (USG) is widely used in both gynaecology and obstetrics. USG has become an indispensable diagnostic tool. Specially in our developing country. Advanced US technologies, such as 3D sonography, provide powerful and highly accurate diagnostic tools. The clinical applications and uses of ultrasound include confirmation of pregnancy including multiple gestation, estimation of gestational age, localization of placenta and monitoring of foetal wellbeing. The others are evaluation of caesarean section scar integrity and post partum haemorrhage. Ultrasound is also useful in prenatal diagnosis of foetal anomaly. The clinical uses of ultrasound in gynaecology include diagnosis of uterine abnormality, ovarian tumour. Ovarian follicles follow up in infertility, diagnosis of ectopic pregnancy, evaluation of abortion case. Detection of pelvic mass \& polycystic ovarian disease is also important. Ultrasound guided FNAC is also an important diagnostic tool.
\end{abstract}

TAJ 2011; 24(2): 152-155

\section{Introduction}

The evolution of diagnostic ultrasonography has been due to the combined efforts of physicists, mechanical and biomedical engineers, computer technologists, researchers and clinical sonographers.

The word ultra means beyond. So ultrasound means something beyond the sound. Ultrasound is a form of energy consisting of mechanically product waves with frequencies above the range of human hearing i.e. more than 20,000 HartZ. Audible sound is upto $20 \mathrm{KHZ}$. Ultrasound is 2-10 MHZ.

Ultrasonography has extended rapidly over the last two decade in world wide. It is a painless, noninvasive, diagnostic tool for almost any pelvic as well as abdominal abnormality, since all structures, normal \& abnormal can usually be demonstrated. It is free of radiation hazard.
Regarding safety of ultrasound, current data indicate that there are no confirmed biologic effect on patients and the use of diagnostic ultrasound evaluation and that the benefits to patients \& fetus. Overall, it is less expensive and within the range of in our poor people.

Ultrasound (US) is widely used in both gynaecology and obstetrics, using high-frequency sound waves to create an image.[1] Standards of practice for conducting pelvic US have been established by scientific societies, and this exam may be performed through transvaginal (TVS) or transabdominal routes,[2,3] but the latter method has not been found to be useful in the evaluation of the female pelvis because of the relatively poor resolution associated with US with the use of lower-frequency ultrasound.[4]

${ }^{1}$ Assistant Professor, Department of Obstetrics \& Gynaecology, Rajshahi Medical College.

${ }^{2}$ Senior Consultant, Dept. of Radiology \& Imaging. Rajshahi Medical College, Rajshahi.

3 Junior Consultant, Gynae \& Obs, UHC, Mohanpur, Rajshahi.

${ }^{4}$ Associate Professor \& Head, Dept. of Radiology \& Imaging. Rajshahi Medical College, Rajshahi 
The history of US in association with gynaecology started in the 1970s, and nowadays the use of US is fundamental in the diagnostic process of many gynaecological pathologies. In particular, pelvic US is the first step for evaluating women with abnormal uterine bleeding where extrauterine pelvic disease is suspected. The indications of US gynaecology include diagnosis of uterine abnormality, ovarian tumour, ovarian follicle development surveillance in infertility work-up, ectopic pregnancy (Fig-1), evaluation of abortion case. Intra-uterine contraceptive device localization, suspected hydatidiform mole or detection of pelvic mass, pelvic inflammatory disease \& polycystic ovarian disease is also can diagnosed by US. A pelvic ultrasound examination will be able to assess the ovaries and exclude the presence of a cyst which may be cancerous.

The indications of US in obstetrics include diagnosis of pregnancy, multiple pregnancy (Fig-2), vaginal bleeding in pregnancy, estimation of gestational age, suspected foetal death, suspected oligohydromnios or polyhydramnios, diagnosis of fetal congenital anomalies, estimation of foetal weight, biophysical profile for foetal well-being, location of the placenta or suspected abruptio placenta .

Interventional use of US include aspiration of ova for in-vitro-fertilization or embryo transfer, aspiration of ovarian cyst, aspiration of drainage of pelvic fluid collection, treatment of ectopic pregnancy by injection of potassium chloride \& methotrexate, early amniocentesis and chorionic villus sampling.

Despite the well-established role of ultrasound in current gynaecological/obstetric practices and patient care, US technology is continuously evolving, and in particular the introduction of 3D US has provided new tools, such as multi-planar display, coronal plane, virtual navigation, tomographic US images and automated volume calculation.

Diagnostic utility of ultrasound guided fine needle aspiration cytology in detection of ovarian tumors, whether it is benign or malignant is also important.

\section{The role of ultrasound}

Ultrasound is considered a safe, non-invasive, accurate and cost-effective imaging technique. It has progressively become an indispensable tool and plays an important role in the care of women. Significant technological development has meant that US can be used to confidently diagnose many common gynaecological pathologies involving the uterus, fallopian tubes and/or the ovary. Nongynaecological pathologies involving the bowel can also be diagnosed with accuracy and will often be seen in the acute gynaecological setting. Ultrasound can be used to triage patients into appropriate treatment protocols, enabling the clinician to avoid surgery in some cases and select the correct surgical approach in others.[5] TVS for assessing uterine pathology has become standard practice in gynaecology; in fact this technique has been proved to detect more pathologies much more accurately than physical examination, and many current protocols for managing women with suspected uterine pathology include a US exam.[2]

In addition, US can be used for extrauterine pathology - most adnexal masses have a typical TVS appearance related to macroscopic pathology (so-called "pattern recognition").[3] For this reason TVS is frequently used as the first imaging technique when extrauterine pelvic disease is suspected. And when there is uncertainty in the evaluation of adnexal masses, the addition of colour Doppler can be useful to identify and grade malignant risk;[3] in particular, malignancy should be suspected when arterial flow is identified in an echogenic mass, whereas benign colour Doppler patterns are considered in the absence of vascular flow or when the only localisation of flow is in the wall of an echogenic mass.[3]

Infertility is a very common problem in our community affecting $10-15 \%$ of couples. Trans Vaginal ultrasound Scan (TVS) remains the most common test and procedure for the fertility investigations as well as the treatment plans. An ultrasound examination may be extremely useful in assessing the causes of infertility as well as assisting in the management of patients once treatment has been started.

There is no need to have an full bladder for this internal scan, which usually does not take more than a few minutes to carry out and no anaesthetic is required either. It helps us to assess the womb structure, lining 
development, ovaries, follicle tracking as well as the blood flow to the pelvic organs.

TVS is an essential test to perform egg collection for IVF/ICSI treatments and a vital tool to assess the well being of the early pregnancy from 6 weeks gestation onwards.

Men may also require the TRS (Trans Rectal ultrasound Scan) if the sperm count shows absence of any sperm to rule out serious testicular or other blockages.

US technology is continuously evolving, and highresolution probes and 3D US probably represent the most important advances. With the introduction of high-resolution vaginal probes TV-US has assumed an important role in assessing the myometrium and the endometrial echo complex. More recently, the development and diffusion of 3D technology has provided further tools to study and analyse these fields. 3D ultrasound can depict lesions not easily diagnosed by conventional 2D ultrasound due to the coronal plane being easily accessible (see Figure 2). A major advantage of 3D ultrasound is the ability to rapidly acquire and store ultrasonographic data. This volume data can then be retrospectively analysed with no loss of information. 3D US also offers new tools such as multiplanar display. Several studies have demonstrated that, with the acquisition of coronal planes, 3D US is extremely accurate in the detection and char-acterisation of congenital uterine anomalies that can be difficult to detect with conventional TVS,[2] with a sensitivity and reproducibility similar to magnetic resonance imaging (MRI). The introduction of 3D US technology has opened up a formidable research field.[2]

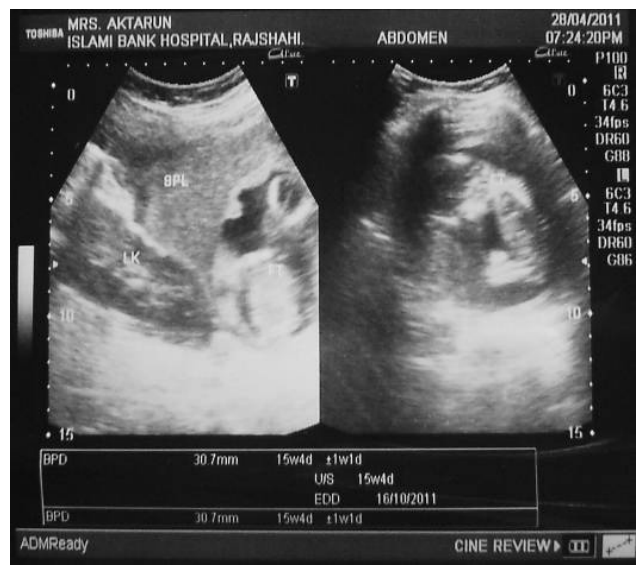

Fig. 1: USG shows an intra-abdominal pregnancy

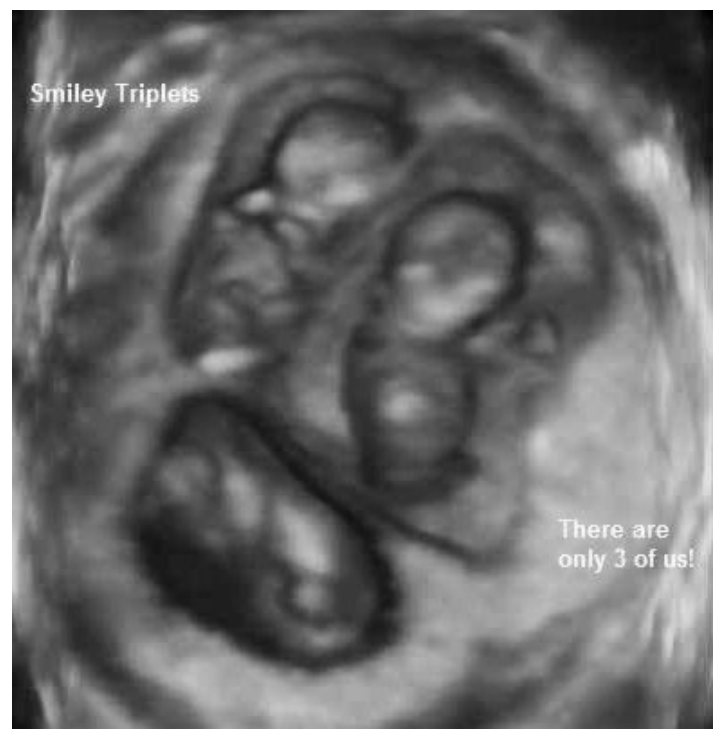

Fig. 2: 3D Ultrasound Triplets at 8 Weeks

It is certainly true that the morphologic evaluation of a fetus is only performed by using USG and that this represents the cornerstone in the evaluation of pregnancy-related complaints,[6] but it is also true that most gynaecological diagnoses are obtained by USG.[7] It is the view of most physicians that USG should not just take place after the history and physical examinations have come up with a working or "differential" diagnosis, but should be considered part of the initial physical examination.[8]

The necessity to reach a high negative predictive value of USG is driving several gynaecological diagnostic processes towards the use of other imaging modalities, in particular computed tomography and MRI,[9,10] even though ultrasonography seems to be the first-line technique of imaging and in some cases the most costeffective.[3] Appropriate training is essential for performing pelvic US.[10] It is a commonly held belief that US exams suffer from a low interobserver agreement,[11] but recent publications have modified this view by indicating that in a US exam it is possible to reach an optimal interobserver agreement by indicating that the most important factor in imaging diagnosis is the observer experience.[12-14] The false idea that everything in this field has been demonstrated and investigated. On the contrary, there are several parts of this discipline where there are "dark" areas - for example, in the detection of pelvic adhesions.[15] 
However, US represent a cornerstone in the study of gynaecological as well as obstetrical pathologies and thanks to rapid technological developments. So, US became a routine part specially in gynaecological visit.

\section{References}

1. Hangiandreou NJ. AAPM/RSNA physics tutorial for residents. Topics in US: B-mode US: basic concepts and new technology. Radiographics 2003; 23:1019-33.

2. Alcazar JL, Galvan R, Garcia-Manero M, Ajossa S, Guerriero S, Melis GB. Transvaginal ultrasound in the diagnosis of uterine pathology. Expert Rev Obstet Gynecol 2008; 3:753-60.

3. Guerriero S, Ajossa S, Gerada M, Virgilio B, Pilloni M, Galvan R, et al. Transvaginal ultrasonography in the diagnosis of extrauterine pelvic diseases. Expert Rev Obstet Gynecol 2008; 3:731-52.

4. Bourne T. Can ovarian masses be characterized using ultrasound? Gynecol Oncol 1993; 51:4-6.

5. Okaro E, Valentin L. The role of ultrasound in the management of women with acute and chronic pelvic pain. Best Pract Res Clin Obstet Gynaecol 2004;18:105-23.

6. Timor-Tritsch IE, Fuchs KM, Monteagudo A, D'alton ME. Performing a fetal anatomy scan at the time of first-trimester screening. Obstet Gynecol 2009; 113:402-7.

7. Enakpene CA, Omigbodun AO, Goecke TW, Odukogbe AT, Beckmann MW. Preoperative evaluation and triage of women with suspicious adnexal masses using risk of malignancy index. J Obstet Gynaecol Res 2009; 35(1):131-8.

8. Goldstein SR. Routine use of office gynecological ultrasound. J Ultrasound Med 2002; 21:489-92.
9. Bazot M, Lafont C, Rouzier R, Roseau G, Thomassin-Naggara I, Daraï E. Diagnostic accuracy of physical examination, transvaginal sonography, rectal endoscopic sonography, and magnetic resonance imaging to diagnose deep infiltrating endometriosis. Fertil Steril 2008; Nov 17. [Epub ahead of print].

10. Bazot M, Daraï E, Nassar-Slaba J, Lafont C, Thomassin-Naggara I. Value of magnetic resonance imaging for the diagnosis of ovarian tumors: a review. J Comput Assist Tomogr 2008; 32:712-23.

11. Opolskiene G, Sladkevicius $P$, Valentin $L$. Ultrasound assessment of endometrial morphology and vascularity to predict endometrial malignancy in women with postmenopausal bleeding and sonographic endometrial thickness. Ultrasound Obstet Gynecol 2007; 30:332-40.

12. Guerriero S, Alcazar JL, Pascual MA, Ajossa S, Gerada M, Bargellini R, et al. Diagnosis of the most frequent benign ovarian cysts: is ultrasonography accurate and reproducible? J Womens Health (Larchmt) 2009; 18:519-27.

13. Guerriero S, Alcazar JL, Pascual MA, Ajossa S, Gerada $M$, Bargellini $R$, et al. Intraobserver and interobserver agreement of greyscale typical ultrasonographic patterns for the diagnosis of ovarian cancer. Ultrasound Med Biol 2008; 34:1711-6.

14. Alcazar JL, Ajossa S, Floris S, Bargellini R, Gerada M, Guerriero S. Reproducibility of endometrial vascular patterns in endometrial disease as assessed by transvaginal power Doppler sonography in women with postmenopausal bleeding. J Ultrasound Med 2006; 25:159-63.

15. Guerriero S, Ajossa S, Garau N, Alcazar JL, Mais $\mathrm{V}$, Melis GB. Diagnosis of pelvic adhesions in patients with endometrioma: the role of transvaginal ultrasonography. Fertil Steril 2009; Apr 13. [Epub ahead of print].
All correspondence to: Ziban Nahar Assistant Professor Department of Obstetrics \& Gynaecology Rajshahi Medical College, Rajshahi, Bangladesh 